\title{
THE HOMOGENIZED EQUATION OF A HETEROGENEOUS REACTION-DIFFUSION MODEL INVOLVING PULSATING TRAVELING FRONTS*
}

\author{
MOHAMMAD EL SMAILY†
}

\begin{abstract}
The goal of this paper is to find the homogenized equation of a heterogeneous reaction-diffusion model in a periodic medium. The solutions of this model are pulsating traveling fronts whose speeds are superior to a parametric minimal speed $c_{L}^{*}$. We first find the homogenized limit of the stationary states which depend on the space variable in many cases. Then, we prove that the pulsating traveling fronts converge to the classical solution $u_{0}:=u_{0}(t, x)$ of a homogeneous reaction-diffusion equation. The homogenized limit $u_{0}$ is also a traveling front whose minimal speed of propagation is given in terms of the harmonic mean of the diffusion and the arithmetic mean of the reaction.
\end{abstract}

Key words. Homogenization, reaction-diffusion, front propagation, heterogeneous media, minimal speed of propagation.

AMS subject classifications. 35B27, 35B45, 35K55, 35K57, 35Q35, 35Q92, 74Q05, 74Q10, 92B05.

\section{Introduction and setting of the problem}

This paper is a continuation of the study of the propagation phenomena of pulsating traveling fronts solving a heterogeneous reaction-diffusion equation. The notion of traveling fronts arose in 1937 in the homogeneous model of Fisher [12] and Kolmogorov, Petrovsky, and Piskunov [17]. This model describes certain population dynamics. In the one-dimensional case, it corresponds to the following equation:

$$
\frac{\partial u}{\partial t}=D \frac{\partial^{2} u}{\partial x^{2}}+u(\mu-\nu u), t>0, x \in \mathbb{R}
$$

The unknown $u=u(t, x)$ is the population density at time $t$ and position $x$, and the positive constant coefficients $D, \mu$, and $\nu$ respectively correspond to the diffusivity (mobility of the individuals), the intrinsic growth rate, and the susceptibility to crowding effects.

Later, many works extended the notion of traveling fronts to the notion of pulsating traveling fronts solving a heterogeneous reaction-advection-diffusion equation in any dimensional space and in general periodic domains (see for example $[1,4,5,24,25,26,27,28,29]$ and [30]). We will recall, after introducing the terms in our problem, the definition of pulsating traveling fronts in the one-dimensional case. The references which were mentioned above can give a detailed and wide description of this notion in higher dimensions and in many general settings.

In this paper, the setting is similar to that in El Smaily, Hamel, and Roques [10]. We consider the parametric heterogeneous reaction-diffusion equation $(L>0$ is the

* Received: December 11, 2010; accepted (in revised version): April 12, 2011. Communicated by Jack Xin.

The author is indebted to the CMU-Portugal program, Center for Nonlinear Analysis (CNA) and Pacific Institute for the Mathematical Sciences (PIMS) for their support during the preparation of this work.

†Carnegie Mellon University, Department of Mathematical Sciences and Center for Nonlinear Analysis, Wean Hall, Pittsburgh, PA, 15213 (elsmaily@andrew.cmu.edu). 
parameter)

$$
\frac{\partial u}{\partial t}=\frac{\partial}{\partial x}\left(a_{L}(x) \frac{\partial u}{\partial x}\right)+f_{L}(x, u), \quad t \in \mathbb{R}, x \in \mathbb{R} .
$$

The diffusion term $a_{L}$ satisfies

$$
a_{L}(x)=a(x / L)
$$

where $a$ is a $C^{2, \alpha}(\mathbb{R})($ with $\alpha>0) 1$-periodic function that satisfies

$$
\exists 0<\alpha_{1}<\alpha_{2}, \forall x \in \mathbb{R}, \alpha_{1} \leq a(x) \leq \alpha_{2} .
$$

The reaction term satisfies $f_{L}(x, \cdot)=f(x / L, \cdot)$, where we assume that $f:=f(x, s)$ : $\mathbb{R} \times \mathbb{R}_{+} \rightarrow \mathbb{R}$ is 1-periodic in $x$, of class $C^{1, \alpha}$ in $(x, s), C^{2}$ in $s$ over $(0, M)$ ( $M$ is defined below at (1.5)), and for each $x \in \mathbb{R}, s \mapsto f(x, s) / s$ is continuous to the right at $s=0$. We set

$$
\mu(x):=\lim _{s \rightarrow 0^{+}} \frac{f(x, s)}{s} \text { and } \mu_{L}(x):=\lim _{s \rightarrow 0^{+}} \frac{f_{L}(x, s)}{s}=\mu\left(\frac{x}{L}\right) .
$$

In biological invasions, $\mu$ stands for the growth rate. Here, $\mu$ may depend on the position $x$. The more favorable the region is, the higher the growth rate $\mu$ is. In this setting, both $a_{L}$ and $f_{L}$ are $L$-periodic in the variable $x$. Furthermore, we assume that:

$$
\left\{\begin{array}{l}
\forall x \in \mathbb{R}, \quad f(x, 0)=0, \\
\exists M \geq 0, \forall s \geq M, \forall x \in \mathbb{R}, \quad f(x, s) \leq 0 .
\end{array}\right.
$$

In the main result of this paper, we need the assumption

$$
\forall x \in \mathbb{R}, \forall s \in(0, M), f(x, s)>0 .
$$

Moreover, to ensure the existence of pulsating traveling fronts, we assume that $f$ satisfies the following condition:

$$
\forall x \in \mathbb{R}, \quad s \mapsto f(x, s) / s \text { is decreasing in } s>0 .
$$

Let, for each $s \in \mathbb{R}$,

$$
g(s):=\int_{0}^{1} f(x, s) d x=<f(\cdot, s)>_{A}
$$

$(<\cdot\rangle_{A}$ stands for the arithmetic mean of a function; see Definition 1.1 below). Then (1.5) and (1.7) yield that $g(0)=0, g(s) \leq 0$ for all $s \geq M$, and $s \mapsto \frac{g(s)}{s}$ is decreasing in $s$.

The stationary states $p(x)$ of (1.2) satisfy the equation

$$
\frac{\partial}{\partial x}\left(a_{L}(x) \frac{\partial p}{\partial x}\right)+f_{L}(x, p)=0, x \in \mathbb{R} .
$$

Under general hypotheses including those of this paper, and in any space dimension, it was proved in [4] that a necessary and sufficient condition for the existence of a 
positive and bounded solution $p$ of (1.8) was the negativity of the principal eigenvalue $\rho_{1, L}$ of the linear operator

$$
\mathcal{L}_{0}: \Phi \mapsto-\left(a_{L}(x) \Phi^{\prime}\right)^{\prime}-\mu_{L}(x) \Phi,
$$

with the periodicity condition that $\Phi(x)$ is $L$-periodic in $x$. In this case, the solution $p$ was also proved to be unique, and therefore $L$-periodic. Actually, it is easy to see that the map $L \mapsto \rho_{1, L}$ is nonincreasing in $L>0$, and even decreasing as long as $a$ is not constant (see the proof of Lemma 3.1 in [10]). Furthermore, $\rho_{1, L} \rightarrow-\int_{0}^{1} \mu(x) d x$ as $L \rightarrow 0^{+}$. In this paper, the assumption (1.6) yields that $\mu(x)$ is positive everywhere and hence

$$
\int_{0}^{1} \mu(x) d x>0
$$

The hypothesis (1.9) guarantees that (for more details about this, see (3.6) below)

$$
\forall L>0, \quad \rho_{1, L}<0 .
$$

Throughout this paper, we set $<\cdot\rangle_{A}$ as follows.

Definition 1.1 (Arithmetic Mean). Let $w: \mathbb{R} \mapsto \mathbb{R}$ be an $l$-periodic function (for some $l>0)$. If $w$ is integrable over its period $[0, l]$, we define the arithmetic mean of $w$ as

$$
<w>_{A}:=\frac{1}{l} \int_{0}^{l} w(x) d x
$$

Now, we recall the definition of pulsating traveling fronts in the one-dimensional case.

Definition 1.2 (Pulsating traveling fronts). A function $u=u(t, x)$ is called a pulsating traveling front propagating from right to left with an effective speed $c \neq 0$, if $u$ is a classical solution of

$$
\left\{\begin{array}{l}
\frac{\partial u}{\partial t}=\frac{\partial}{\partial x}\left(a_{L}(x) \frac{\partial u}{\partial x}\right)+f_{L}(x, u), \quad t \in \mathbb{R}, x \in \mathbb{R}, \\
\forall k \in \mathbb{Z}, \forall(t, x) \in \mathbb{R} \times \mathbb{R}, \quad u\left(t+\frac{k L}{c}, x\right)=u(t, x+k L), \\
0 \leq u(t, x) \leq p_{L}(x), \\
\lim _{x \rightarrow-\infty} u(t, x)=0 \text { and } \lim _{x \rightarrow+\infty} u(t, x)-p_{L}(x)=0,
\end{array}\right.
$$

where the above limits hold locally in $t$.

This definition was given in any space dimension in [1] and [27] whenever the stationary state $p_{L} \equiv 1$ and in [5] whenever $p_{L} \not \equiv 1$.

For each $L>0$, assuming (1.3) on the diffusion $a,(1.5),(1.7)$, and (1.9) on the nonlinearity $f$, the results of [5] yield that there exists $c_{L}^{*}>0$ such that pulsating traveling fronts of (1.11) which propagate with a speed $c$ exist if and only if $c \geq c_{L}^{*}$. The value $c_{L}^{*}$ is called the minimal speed of propagation. We refer to $[2,3,8,9,10,11$, $15,18,19,13,20,21,22,23,26,31]$ for further results on the existence and properties of the minimal speed in the KPP case. We mention that the limit of the minimal 
wave speeds was considered in [18] but there are no results about the homogenized equation.

In El Smaily, Hamel, Roques [10], the homogenized speed was found by calculating the limit of $c_{L}^{*}$ as $L \rightarrow 0^{+}$. Precisely, Theorem 2.1 in [10] yields that

$$
\lim _{L \rightarrow 0^{+}} c_{L}^{*}=2 \sqrt{<a>_{H}<\mu>_{A}},
$$

where

$$
<\mu>_{A}=\int_{0}^{1} \mu(x) d x \text { and }<a>_{H}=\left(\int_{0}^{1}(a(x))^{-1} d x\right)^{-1}=<a^{-1}>_{A}^{-1}
$$

denote the arithmetic mean of $\mu$ and the harmonic mean of $a$ over the interval $[0,1]$. This result was proved rigorously and it generalized the formal and numerical results of $[16]$.

Having (1.12), there arise several questions about the homogenized equation of (1.11) and the nature of the homogeneous limit of the pulsating traveling fronts $u_{L}$ and the type of convergence of $\left\{u_{L}\right\}_{L}$ as the periodicity parameter $L \rightarrow 0^{+}$. The main goal of this work is to answer these questions.

In this paper, some difficulties arise in finding $H_{l o c}^{1}(\mathbb{R} \times \mathbb{R})$ estimates, independent of $L$, for a sequence of pulsating traveling fronts $\left\{u_{L}\right\}_{L}$ and for the corresponding sequence $\left\{a_{L} \frac{\partial u_{L}}{\partial x}\right\}_{L}$. In fact, each pulsating traveling front $u_{L}$ satisfies a sort of $(t, x)$-periodicity (see the second line of (1.11)). This fact makes the procedure leading to the desired estimates indirect. Another difficulty comes from the dependence of the stationary states $p_{L}$ on the space variable $x$. This is due to the choice of a wider class of heterogeneous nonlinearities in the present work. We mention that the situation becomes simpler if we assume that there is a positive value $s_{0}$ such that $f\left(x, s_{0}\right)=0$ for any $x \in \mathbb{R}$ and that $f(x, s)>0$ in $\mathbb{R} \times\left(0, s_{0}\right)$. Indeed, this yields that $p_{L} \equiv s_{0}$ for all $L>0$ (see [4] and [5] for more details). One of the techniques that we use in this present work appears in Step 3 of the proof of Theorem 2.3. It consists of deriving the reaction-diffusion equation with respect to the time variable and then getting estimates on the functions $w_{L}:=\partial u_{L} / \partial t$ and $v_{L}:=a_{L}(x) \partial u_{L} / \partial x$.

\section{Main results}

Before going further in this section, we recall that the function $g$ defined by

$$
\forall s \in \mathbb{R}, g(s):=\int_{0}^{1} f(x, s) d x
$$

satisfies $g(0)=0$. Referring to Definition 1.1, one can rewrite

$$
\forall s \in \mathbb{R}, g(s)=<\cdot, s>_{A} .
$$

Moreover, (1.9) yields that

$$
g^{\prime}(0)=\lim _{s \rightarrow 0+} \int_{0}^{1} \frac{f(x, s)}{s}=\int_{0}^{1} \mu(x) d x>0 .
$$

Owing to (1.5) and (1.7), the map $s \mapsto \frac{g(s)}{s}$ is decreasing and $g(s) \leq 0$ for all $s \geq M$. As a consequence, we let $p_{0}$ stand for the only root of $g(s)$ which is strictly positive. 
The following lemma gives several convergence results of the sequence $\left\{p_{L}\right\}_{L>0}$ of stationary states as $L \rightarrow 0^{+}$:

Lemma 2.1 (The homogenized stationary state at $+\infty$ ). Assume that the diffusion $a=a(x)$ satisfies (1.3) and the nonlinearity $f$ satisfies (1.5) and (1.7) together with $\int_{0}^{1} \mu(x) d x>0$. Let $\left\{L_{n}\right\}_{n \in \mathbb{N}}$ be a sequence of positive real numbers in $(0,1)$ such that $L_{n} \rightarrow 0^{+}$as $n \rightarrow+\infty$. Let $p_{0}$ denote the unique positive zero of the function $g(s):=<f(\cdot, s)>_{A}$ (see Definition 1.1 and the explanation above about $\left.p_{0}\right)$. For each $n \in \mathbb{N}$, the function $p_{L_{n}}:=p_{L_{n}}(x)$ denotes the unique stationary state at $+\infty$ of the Equation (1.8) with $L=L_{n}$. Then

i) The sequence $\left\{p_{L_{n}}\right\}_{n \in \mathbb{N}}$ is bounded in $H_{l o c}^{1}(\mathbb{R})$.

ii) $p_{L_{n}} \rightarrow p_{0}$ in $H_{\text {loc }}^{1}(\mathbb{R})$ weak and $p_{L_{n}} \rightarrow p_{0}$ in $L_{\text {loc }}^{2}(\mathbb{R})$ strong as $n \rightarrow+\infty$.

iii) $p_{L_{n}} \rightarrow p_{0}$ in $C_{\text {loc }}^{0, \delta}(\mathbb{R})$ as $n \rightarrow+\infty$ for all $0 \leq \delta<1 / 2$.

REMARK 2.2. We mention that the assumption (1.6) is not needed in Lemma 2.1. Concerning the nonlinearity $f$, we assume that $\int_{0}^{1} \mu(x) d x>0$ in order to guarantee the existence and the uniqueness of the stationary state $p_{L}$ solving (1.8) for each $L>0$. The results of Lemma 2.1 hold in the cases where the sign of $\mu$ may be positive in some regions (favorable regions) and negative in others (unfavorable regions) provided that $\int_{0}^{1} \mu(x) d x>0$.

We announce the homogenized equation of (1.11) and some convergence results of $\left\{u_{L}\right\}_{L>0}$ as $L \rightarrow 0^{+}$in the following theorem.

Theorem 2.3 (Homogenized equation after normalization). Assume that the diffusion a satisfies (1.3) and the reaction $f$ satisfies (1.5-1.6) and (1.7). Let $\left\{L_{n}\right\}_{n \in \mathbb{N}}$ be a sequence of positive numbers in $(0,1)$ such that $L_{n} \rightarrow 0^{+}$as $n \rightarrow+\infty$. For each $n \in \mathbb{N}$, let $\left(c_{L_{n}}, u_{L_{n}}\right)$ be the unique pulsating traveling front solving (1.11) for $L=L_{n}$, propagating with the speed $c_{L_{n}} \geq c_{L_{n}}^{*}$, and satisfying the normalization

$$
\forall 0<L_{n} \leq L_{0}, \quad \iint_{(0,1) \times(0,1)} u_{L_{n}}(t, x) d t d x=\frac{p_{0}}{2}
$$

(a justification of this normalization is given in Step 2 of the proof). Assume that $\left\{c_{L_{n}}\right\}_{n \in \mathbb{N}}$ converges and call $c:=\lim _{n \rightarrow+\infty} c_{L_{n}}$. On the other hand, let $u_{0}(t, x)=U_{0}(x+$ ct) denote the traveling front propagating from right to left with the speed $c$, normalized by

$$
\iint_{(0,1)^{2}} u_{0}(t, x) d t d x=\frac{p_{0}}{2}
$$

and which is a classical solution of the homogeneous reaction-diffusion equation

$$
\frac{\partial u_{0}}{\partial t}=<a>_{H} \frac{\partial^{2} u_{0}}{\partial x^{2}}+g\left(u_{0}\right) \text { in } \mathbb{R} \times \mathbb{R},
$$

with $U_{0}(-\infty)=0$ and $U_{0}(+\infty)=p_{0}$ in $C_{l o c}^{2}(\mathbb{R})$. Then,

$$
u_{L_{n}} \rightarrow u_{0} \text { as } n \rightarrow+\infty \text { in } H_{\text {loc }}^{1}(\mathbb{R} \times \mathbb{R}) \text { weak and in } L_{\text {loc }}^{2}(\mathbb{R} \times \mathbb{R}) \text { strong. }
$$


The above theorem holds for pulsating traveling fronts with speeds $c_{L} \in\left[c_{L}^{*},+\infty\right)$. Physically and biologically, the fronts propagating with a speed $c_{L}^{*}$ (the minimal speed) are the most interesting. Due to [10], any sequence $\left\{c_{L_{n}}^{*}\right\}_{n}$ will converge to $2 \sqrt{\langle a\rangle_{H}} \sqrt{\langle\mu\rangle_{A}}$ as $L_{n} \rightarrow 0^{+}$. However, for the sake of completeness, we announced Theorem 2.3 for any sequence $c_{L} \geq c_{L}^{*}$ provided that the chosen sequence of speeds converges to $c$. Here, it is important to recall the existence results of pulsating traveling fronts in [5] together with the uniqueness results, up to a shift in the time variable, proved by Hamel and Roques [14]. In this present setting, this reads as: for each $c_{L} \geq c_{L}^{*}$, there exists a pulsating traveling front $u_{L}(t, x):=\varphi_{L}\left(x+c_{L} t, x\right)$, and any other pulsating traveling front with the same speed should have the form $u_{L}(t+\sigma, x)$ for some shift $\sigma \in \mathbb{R}$. Of course, one has to take the dependence of the shifts on $L$ into consideration. In this context, we have the following remark.

Remark 2.4. In Theorem 2.3, the normalization (2.2) of the sequence $\left\{u_{L_{n}}\right\}_{n}$ is to guarantee the existence and uniqueness of the limit $u_{0}$ in the effective Equation (2.4). The estimates done in Step 3 of the theorem's proof do not require the normalization. For more details, we refer the reader to the proof of the theorem and especially Step 2 , which deals with the normalization issue, and Step 4, which is the passage to the limit. On the other hand, if one picks a sequence of speeds $c_{L} \rightarrow c>\lim _{L \rightarrow 0^{+}} c_{L}^{*}$, Step 2 of the proof yields that there will exist a normalized sequence $\left\{u_{L_{n}}\right\}_{n}$ satisfying (2.2) and then the convergence result (2.5) will still hold.

The homogenization problem would be very interesting in multi-dimensions. Estimates for the multi-dimensional problem in cylindrical type domains could be obtained in a similar approach to the one we use in the proof of Theorem 2.3, but they are not enough to pass to the limit as $L \rightarrow 0^{+}$. One needs to get further estimates on higher order derivatives of the sequence $u_{L}$. Furthermore, the homogenized speed of such problems was proved rigorously only in the one-dimensional case in [10]. A first step to obtain the homogenized equation in higher dimensions is to homogenize the minimal speeds. This is the subject of a forthcoming paper by the author.

We lastly mention that other homogenization results were found by Caffarelli, Lee and Mellet $[6,7]$ in the case of combustion-type nonlinearities.

Remark 2.5. From the above theorem, we can recover the sharp lower bound of $\liminf c_{L}^{*}$ which was proved in [10]. That sharp lower bound is given by

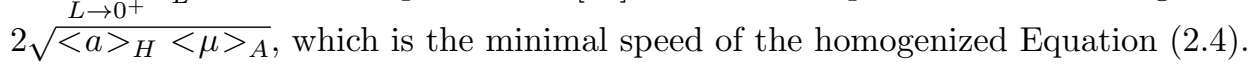

\section{Proofs of the announced results}

Proof of Lemma 2.1. The proof of Lemma 2.1 will be divided into three steps:

Step 1: Convergence to a constant limit $p_{*}$. Under the assumptions of Lemma 2.1 on $f$, it follows from [4] that for each $L>0$, the function $p_{L}$ solving the equation

$$
\left(a_{L}(x) p_{L}^{\prime}\right)^{\prime}+f_{L}\left(x, p_{L}\right)=0, x \in \mathbb{R}
$$

is unique, positive, $L$-periodic, and

$$
\forall L>0, \forall x \in \mathbb{R}, 0<p_{L}(x) \leq M,
$$

where $M$ is the constant appearing in (1.5).

One can directly conclude from above that the sequence $\left\{p_{L_{n}}\right\}_{n \in \mathbb{N}}$ is bounded in $L_{\text {loc }}^{2}(\mathbb{R})$. Now, we fix $L>0$, multiply the Equation (3.1) by $p_{L}$, and then integrate by 
parts over any interval of the form $[-k L, k L]$ where $k \in \mathbb{N}$. Owing to the $L$-periodicity of $p_{L}$, we get

$$
\forall L>0, \forall k \in \mathbb{N}, \quad \int_{-k L}^{k L} a\left(\frac{x}{L}\right)\left(p_{L}^{\prime}\right)^{2} d x=L \int_{-k}^{k} f\left(x, p_{L}(L x)\right) d x .
$$

Consider the values of $L$ included in the interval $(0,1)$ and let $\mathcal{K}$ be any compact interval of $\mathbb{R}$. For each $L>0$, we denote

$$
k_{L}=\left[\frac{|\mathcal{K}|}{2 L}\right]+1 \in \mathbb{N}
$$

where $|\mathcal{K}|$ stands for the Lebesgue measure of the interval $\mathcal{K}$ and [.] stands for the integer part of a real number. One consequently has $|\mathcal{K}| \leq 2 k_{L} L \leq|\mathcal{K}|+2 L$ and $\mathcal{K} \subseteq$ $\left[-k_{L} L+m L, k_{L} L+m L\right]$ for some integer $m \in \mathbb{Z}$ depending on $\mathcal{K}$ and $L$.

Owing to the $L$-periodicity of $f_{L}, a_{L}$, and $p_{L}$ with respect to $x$, together with the assumption (1.3) on the diffusion $a$, and using (3.2), we obtain

$$
\forall k \in \mathbb{N}, \int_{-k L+m L}^{k L+m L} a\left(\frac{x}{L}\right)\left(p_{L}^{\prime}\right)^{2} d x=\int_{-k L}^{k L} a\left(\frac{x}{L}\right)\left(p_{L}^{\prime}\right)^{2} d x=L \int_{-k}^{k} f\left(x, p_{L}(L x)\right) d x .
$$

Consequently, for any compact interval $\mathcal{K}$ in $\mathbb{R}$, we have

$$
\forall 0<L \leq 1, \quad \int_{\mathcal{K}}\left(p_{L}^{\prime}\right)^{2} d x \leq C(\mathcal{K})
$$

where $C(\mathcal{K}):=\frac{|\mathcal{K}|+2}{\alpha_{1}} \max _{(x, s) \in[0,1] \times[0, M]}|f(x, s)|$ is a positive constant independent of $L$ and depending on the size of the compact $\mathcal{K}$. In other words, the sequence $\left\{p_{L_{n}}\right\}_{n \in \mathbb{N}}$ is bounded in $H^{1}(\mathcal{K})$ for any compact $\mathcal{K} \subset \mathbb{R}$ and this completes the proof of part i) of the Lemma.

Furthermore, we can conclude that there exists $p_{*} \in H_{l o c}^{1}(\mathbb{R})$ such that, up to extraction of a subsequence, $p_{L_{n}} \rightarrow p_{*}$ in $H_{l o c}^{1}(\mathbb{R})$ weak and $p_{L_{n}} \rightarrow p_{*}$ in $L_{l o c}^{2}(\mathbb{R})$ strong as $n \rightarrow+\infty$.

Using Sobolev injections, we have $H^{1}(\mathcal{K})$ is embedded in $C^{0,1 / 2}(\mathcal{K})$. Thus, the sequence $\left\{p_{L_{n}}\right\}_{n \in \mathbb{N}}$ is bounded in $C^{0,1 / 2}(\mathcal{K})$. Compact embeddings (Schauder's estimates) yield that one can extract a subsequence, say $\left\{p_{L_{n}}\right\}_{n}$ without loss of generality, that converges in $C_{\text {loc }}^{0, \delta}(\mathbb{R})$ for each $0 \leq \delta<1 / 2$. As a subsequence $\left\{p_{L_{n}}\right\}_{n}$ should also converge to $p_{*}$ in $H_{l o c}^{1}(\mathbb{R})$ weak and $p_{L_{n}} \rightarrow p_{*}$ in $L_{l o c}^{2}(\mathbb{R})$ strong as $n \rightarrow+\infty$. Consequently, for each $0 \leq \delta<1 / 2$ there is a subsequence $p_{L_{n}} \rightarrow p_{*}$, as $n \rightarrow+\infty$, in $C_{\text {loc }}^{0, \delta}(\mathbb{R})$. But since each function $p_{L_{n}}$ is $L_{n}$-periodic (with $L_{n} \rightarrow 0^{+}$as $n \rightarrow+\infty$ ), it follows from Arzela-Ascoli theorem that $p_{*}$ has to be constant over $\mathbb{R}$.

Step 2: The constant limit $p_{*}$ is positive. To achieve this goal, we will compare the stationary states $p_{L}$ with the principal eigenfunctions $\Phi_{L}$ of the eigenvalue problem

$$
\mathcal{L}_{0} \Phi_{L}:=-\left(a_{L}(x) \Phi_{L}^{\prime}\right)^{\prime}-\mu_{L}(x) \Phi_{L}=\rho_{1, L} \Phi_{L} \text { in } \mathbb{R},
$$

which are $L$-periodic and positive in $\mathbb{R}$.

First, we divide (3.5) by $\Phi_{L}$ and then we integrate by parts over $[0, L]$. It then follows from the $L$-periodicity of $\Phi_{L}$ and the coefficients of $\mathcal{L}_{0}$ that

$$
\forall L>0, \quad-\frac{1}{L} \int_{0}^{L} a_{L}\left(\frac{\Phi_{L}^{\prime}}{\Phi_{L}}\right)^{2}-\int_{0}^{1} \mu(x) d x=\rho_{1, L} .
$$


Hence,

$$
\forall L>0, \quad \rho_{1, L} \leq \rho_{1}:=-\int_{0}^{1} \mu(x) d x<0 .
$$

Next, due to the uniqueness up to multiplication by a nonzero constant of $\Phi_{L}$, we can assume that $\left\|\Phi_{L}\right\|_{\infty}=1$ for every $L \in \mathbb{R}$. Since the function $f(x, s)$ is 1-periodic in $x$ and of class $C^{1}$ on $\mathbb{R} \times \mathbb{R}^{+}$, one can then find $\varepsilon_{0}>0$ such that

$$
\forall 0 \leq s \leq \varepsilon_{0}, \forall x \in \mathbb{R}, f(x, s)-\mu(x) s \geq \frac{\rho_{1}}{2} s .
$$

Having $0<\varepsilon_{0} \Phi_{L} \leq \varepsilon_{0}$, we get from (3.6) and (3.7) that

$$
\begin{aligned}
-\left(a_{L} \varepsilon_{0} \Phi_{L}^{\prime}\right)^{\prime}-f\left(\frac{x}{L}, \varepsilon_{0} \Phi_{L}\right) & =\rho_{1, L} \varepsilon_{0} \Phi_{L}+\varepsilon_{0} \mu_{L}(x) \Phi_{L}-f\left(\frac{x}{L}, \varepsilon_{0} \Phi_{L}\right) \\
& \leq \rho_{1} \varepsilon_{0} \Phi_{L}-\frac{\rho_{1}}{2} \varepsilon_{0} \Phi_{L} \\
& =\frac{\rho_{1}}{2} \varepsilon_{0} \Phi_{L}<0 \mathrm{in} \mathbb{R},
\end{aligned}
$$

for all $L>0$.

Let us now fix any $L>0$ and, for simplicity, denote

$$
\psi_{L}:=\varepsilon_{0} \Phi_{L} .
$$

We recall that the functions $p_{L}$ and $\psi_{L}=\varepsilon_{0} \Phi_{L}$ are both positive and $L$-periodic. Hence, we can define

$$
\gamma^{*}:=\sup \left\{\gamma>0, p_{L}>\gamma \psi_{L}\right\} \geq 0 .
$$

Assume to the contrary that $\gamma^{*}<1$. From the assumption (1.7), we have $f\left(x, \gamma^{*} \psi_{L}\right)>$ $\gamma^{*} f\left(x, \psi_{L}\right)$ for all $x \in \mathbb{R}$. Referring to (3.8), the following inequality then holds

$$
-\left(a_{L} \gamma^{*} \psi_{L}^{\prime}\right)^{\prime}-f\left(\frac{x}{L}, \gamma^{*} \psi_{L}\right)<0 \text { in } \mathbb{R} .
$$

Set $z:=p_{L}-\gamma^{*} \psi_{L}$. Then $z \geq 0$, and there exists a sequence $x_{n} \in \mathbb{R}$ such that $z\left(x_{n}\right) \rightarrow$ 0 as $n \rightarrow+\infty$ (by definition of $\gamma^{*}$ ). Owing to the periodicity of $z$, one can then assume that $x_{n} \in[0, L]$. Hence, up to extraction of some subsequence, $x_{n} \rightarrow \bar{x} \in[0, L]$. From continuity, $z(\bar{x})=0$. Besides, it follows from (3.1) and (3.9) that there exists a continuous function $b=b(x)$ such that the nonnegative function $z$ satisfies

$$
\left(a_{L} z^{\prime}\right)^{\prime}+b(x) z<0 \text { in } \mathbb{R} .
$$

The strong maximum principle implies that $z \equiv 0$; and hence, $p_{L} \equiv \gamma^{*} \psi_{L}$. This contradicts with (3.9). Consequently, the assumption that $\gamma^{*}<1$ is false; and thus, $p_{L} \geq \gamma^{*} \psi_{L} \geq \psi_{L}=\varepsilon_{0} \Phi_{L}$ in $\mathbb{R}$. One then concludes that

$$
\forall L>0, \quad \max _{x \in \mathbb{R}} p_{L}(x)=\max _{x \in[0, L]} p_{L}(x) \geq \varepsilon_{0}\left\|\Phi_{L}\right\|_{\infty}=\varepsilon_{0} .
$$

On the other hand, the constant limit $p_{*}$ to which the $L_{n}$-periodic functions $p_{L_{n}}$ converge uniformly on every compact subset of $\mathbb{R}$ as $n \rightarrow+\infty\left(L_{n} \rightarrow 0^{+}\right)$satisfies $p_{*} \geq \liminf _{n \rightarrow+\infty} \max _{x \in \mathbb{R}} p_{L_{n}}(x)$. Therefore, $p_{*} \geq \varepsilon_{0}>0$.

Step 3: The constant limit $p_{*}$ is equal to $p_{0}$. For each $L>0$, we call

$$
q_{L}(x)=a_{L}(x) p_{L}^{\prime}(x), x \in \mathbb{R} .
$$


Equation (3.1) can be rewritten as

$$
\forall L>0, q_{L}^{\prime}+f\left(\frac{x}{L}, p_{L}\right)=0 \text { in } \mathbb{R} .
$$

Consider any compact interval $\mathcal{K}$ of $\mathbb{R}$ and, for each $L$, let $k_{L}>0$ be the integer defined at (3.3). From Equation (3.10) one has

$$
\forall 0<L<1, \int_{-k_{L} L}^{k_{L} L}\left(q_{n}^{\prime}\right)^{2}=L \int_{-k_{L}}^{k_{L}} f^{2}\left(x, p_{L}(L x)\right) d x .
$$

Also, we have $0<p_{L} \leq M$ for all $L>0$, where $M$ is the constant appearing in (1.5). Thus, for each compact interval $\mathcal{K}$ of $\mathbb{R}$, there exists a constant $C_{1}(\mathcal{K}):=$ $(|\mathcal{K}|+2) \max _{[0,1] \times[0, M]}\left|f^{2}(x, s)\right|$, which depends only on $\mathcal{K}$, such that

$$
\forall 0<L<1, \int_{\mathcal{K}}\left(q_{L}^{\prime}\right)^{2}(x) d x \leq C_{1}(\mathcal{K})
$$

Having $\left\{L_{n}\right\}_{n}$ as a sequence of positive numbers in $(0,1)$ such that $L_{n} \rightarrow 0^{+}$as $n \rightarrow$ $+\infty$, we write $p_{n}=p_{L_{n}}$ and $q_{n}=q_{L_{n}}$. The assumption (1.3) together with (3.4) yield that $\left\{q_{n}\right\}_{n}$ is bounded in $L^{2}(\mathcal{K})$. Finally, the sequence is $\left\{q_{n}\right\}_{n}$ is bounded in $H_{l o c}^{1}(\mathbb{R})$. Arguing as in Step 1, we can conclude that there exists a constant $q_{0}$ such that $q_{n} \rightarrow q_{0}$ in $H_{l o c}^{1}(\mathbb{R})$ weak, $q_{n} \rightarrow q_{0}$ in $L_{l o c}^{2}(\mathbb{R})$ strong, and $q_{n} \rightarrow q_{0}$ in $C_{\text {loc }}^{0, \delta}(\mathbb{R})$ for all $0 \leq \delta<1 / 2$. However, $f\left(\frac{x}{L_{n}}, p_{n}\right) \rightarrow g\left(p_{*}\right)$ in $L^{\infty}(\mathbb{R})$ weak-* as $n \rightarrow+\infty$. Passing to the limit as $n \rightarrow+\infty$ in Equation (3.10) (where $L=L_{n}$ ) implies that $g\left(p_{*}\right)=0$. Referring to the properties of the function $g$ which are mentioned at the beginning of Section 3 and owing to the positivity of the constant $p_{*}$, we conclude that $p_{*}=p_{0}$. Eventually, this completes the proof of Lemma 2.1.

Proof of Theorem 2.3. This proof will be done in four steps.

Step 1: Recalling the lower bound for the speeds which proves that $c>0$. From the results of [5], each pulsating traveling front $u_{L_{n}}$ exists if and only if $c_{L_{n}} \geq c_{L_{n}}^{*}$. Moreover, for each $L>0$, the minimal speed $c_{L}^{*}$ is positive and, from [5] (see also [3] in the case when $p \equiv 1$ ), it is given by the variational formula

$$
c_{L}^{*}=\min _{\lambda>0} \frac{k(\lambda, L)}{\lambda}=\frac{k\left(\lambda_{L}^{*}, L\right)}{\lambda_{L}^{*}},
$$

where $\lambda_{L}^{*}>0$ and $k(\lambda, L)$ (for each $\lambda \in \mathbb{R}$ and $L>0$ ) denotes the principal eigenvalue of the problem

$$
\left(a_{L} \phi_{\lambda, L}^{\prime}\right)^{\prime}+2 \lambda a_{L} \phi_{\lambda, L}^{\prime}+\lambda a_{L}^{\prime} \phi_{\lambda, L}+\lambda^{2} a_{L} \phi_{\lambda, L}+\mu_{L} \phi_{\lambda, L}=k(\lambda, L) \phi_{\lambda, L} \text { in } \mathbb{R},
$$

with $L$-periodicity conditions. In (3.13), $\phi_{\lambda, L}$ denotes a principal eigenfunction, which is of class $C^{2, \alpha}(\mathbb{R})$, positive, $L$-periodic and unique up to multiplication by a positive constant. In Section 3 of [10], the author proved with Hamel and Roques that the minimal speeds $c_{L_{n}}^{*}$ satisfy

$$
c:=\lim _{n \rightarrow+\infty} c_{L_{n}} \geq \liminf _{n \rightarrow+\infty} c_{L_{n}}^{*} \geq 2 \sqrt{\alpha_{1}<\mu>_{A}}>0 .
$$

This gives a sharp lower bound for the sequence $\left\{c_{L_{n}}^{*}\right\}$. 
Step 2: Normalization of $u_{L}$ and recalling standard change of variables. For any $L>0$, consider a speed $c_{L} \geq c_{L}^{*}$ and let $W_{L}(t, x)$ be an arbitrarily chosen pulsating traveling front for (1.11) propagating with speed $c_{L}$. We know from Hamel, Roques [14] that the family of pulsating traveling fronts with a speed $c_{L}$ is then of the form $\left\{W_{L, \sigma}\right\}_{\sigma \in \mathbb{R}}$, where

$$
W_{L, \sigma}(t, x)=W_{L}(t+\sigma, x), \quad(t, x) \in \mathbb{R}^{2} .
$$

This is the uniqueness up to a shift in the time variable of traveling fronts with the same speed $c_{L}$. We want to pick one front of this family and use it in the homogenization procedure. Once the appropriate normalization is picked, we will drop the index $\sigma$ and continue the proof with the notation $u_{L}$ (or $\varphi_{L}$ ) for our front. We start by recalling the standard change of variables

$$
\forall L>0, \forall \sigma \in \mathbb{R}, W_{L, \sigma}(t, x):=\varphi_{L, \sigma}\left(x+c_{L} t, x\right)=\varphi_{L, \sigma}(s, x),(t, x) \in \mathbb{R} \times \mathbb{R} .
$$

The $(t, x)$-periodicity (or the definition of the speed)

$$
W_{L, \sigma}\left(t, x+\frac{k L}{c_{L}}\right)=W_{L}(t, x+k), \text { for all } k \in \mathbb{Z},
$$

came, in [5] for example, by the construction of $\varphi_{L, \sigma}$ (for any shift $\sigma \in \mathbb{R}$ ) which is $L$-periodic with respect to the spatial variable $x$ and solves the equation

$$
\partial_{x}\left(a_{L} \partial_{x} \varphi_{L, \sigma}\right)+a_{L} \partial_{s s} \varphi_{L, \sigma}+\partial_{x}\left(a_{L} \partial_{s} \varphi_{L, \sigma}\right)+\partial_{s}\left(a_{L} \partial_{x} \varphi_{L, \sigma}\right)-c_{L} \partial_{s} \varphi_{L}+f_{L}\left(x, \varphi_{L}\right)=0,
$$

for all $(s, x) \in \mathbb{R} \times \mathbb{R}$. This is (1.11) in terms of the new function $\varphi_{\sigma, L}$. Furthermore, one has $\varphi_{L, \sigma}(s, x) \rightarrow 0$ as $s \rightarrow-\infty$ and $\varphi_{L, \sigma}(s, x) \rightarrow p(x)$ as $s \rightarrow+\infty$ in $C^{2}(\mathbb{R})$ (in fact, this follows mainly from the standard elliptic estimates and from the periodicity of $\varphi_{L, \sigma}$ with respect to $x$ ). As a consequence, it was proved in [5] that

$$
\forall L>0, \sigma \in \mathbb{R}, \lim _{t \rightarrow-\infty} W_{L, \sigma}(t, x)=0 \text { and } \lim _{t \rightarrow+\infty} W_{L, \sigma}(t, x)=p(x) \text { in } C_{l o c}^{2}(\mathbb{R}) .
$$

Then, the $(t, x)$-periodicity of the functions $W_{L, \sigma}$ leads to the limiting conditions $\lim _{x \rightarrow-\infty} W_{L, \sigma}(t, x)=0$ and $\lim _{x \rightarrow+\infty} W_{L, \sigma}(t, x)=p(x)$ locally in $t$.

Now, we define the function

$$
\forall L>0, I_{L}(\sigma)=\int_{(0,1)^{2}} W_{L}(t+\sigma, x) d t d x,
$$

which is continuous over $\mathbb{R}$. We recall that for each $L>0$, the function $W_{L}$ is increasing in the first variable (time), hence $I_{L}$ is increasing in $\sigma \in \mathbb{R}$. Also, $I_{L}$ satisfies $\lim _{\sigma \rightarrow-\infty} I_{L}(\sigma)=0$ and

$$
\lim _{\sigma \rightarrow+\infty} I_{L}(\sigma)=\int_{0}^{1} p_{L}(x) d x \geq \min _{x \in \mathbb{R}} p_{L}(x)>\frac{p_{0}}{2}>0 \text { for all } 0<L \leq L_{0},
$$

for some $L_{0}$ small enough which exists by the $L$-periodicity of $p_{L}$ and the uniform convergence of $p_{L}$ to $p_{0}$ as $L \rightarrow 0^{+}$. It is important here to notice that $L_{0}$ is determined only by the uniform convergence of the stationary states $p_{L}(x)$ to $p_{0}$ which is the unique positive root of $g$. The stationary state $p_{L}$ is the same, even if one considers 
different shifts in the time variable of the function $u_{L}(t, x)$ (see [5] for more details). Now, we use the continuity and monotonicity of $I_{L}$ with respect to $\sigma$ to conclude that

$$
\forall 0<L \leq L_{0}, \exists \text { unique } \sigma_{L} \in \mathbb{R}, \iint_{(0,1) \times(0,1)} W_{L}\left(t+\sigma_{L}, x\right) d t d x=\frac{p_{0}}{2} .
$$

Conclusion. For each $0<L \leq L_{0}$, given a speed $c_{L} \geq c_{L}^{*}$, one can then denote by

$$
u_{L}(t, x):=W_{L}\left(t+\sigma_{L}, x\right)
$$

$\left(\sigma=\sigma_{L}\right)$ the unique pulsating traveling front solving (1.11) and propagating with a speed $c_{L} \geq c_{L}^{*}$ that satisfies the normalization

$$
\forall 0<L \leq L_{0}, \quad \iint_{(0,1) \times(0,1)} u_{L}(t, x) d t d x=\frac{p_{0}}{2} .
$$

In the same context $\left(\sigma=\sigma_{L}\right)$, we set the corresponding $\varphi_{L}$ as

$$
u_{L}(t, x):=\varphi_{L}(x+c t, x)=\varphi(s, x)
$$

which, from (3.14), satisfies the equation

$$
\partial_{x}\left(a_{L} \partial_{x} \varphi_{L}\right)+a_{L} \partial_{s s} \varphi_{L}+\partial_{x}\left(a_{L} \partial_{s} \varphi_{L}\right)+\partial_{s}\left(a_{L} \partial_{x} \varphi_{L}\right)-c_{L} \partial_{s} \varphi_{L}+f_{L}\left(x, \varphi_{L}\right)=0,
$$

for all $(s, x) \in \mathbb{R} \times \mathbb{R}$.

Step 3: Boundedness of $\left\{u_{L_{n}}\right\}_{n}$ and $\left\{a_{L_{n}} \partial_{x} u_{L_{n}}\right\}_{n}$ in $H_{\text {loc }}^{1}(\mathbb{R} \times \mathbb{R})$. To simplify notations, we drop the $n$ and consider the family $\left\{\left(c_{L}, u_{L}\right)\right\}_{0<L<1}$ of pulsating traveling fronts solving (1.11), satisfying the normalization (3.17) and such that $0<\underline{c} \leq c_{L} \leq \bar{c}$ for all $0<L<1$, where $\underline{c}$ and $\bar{c}$ are two positive constants. We mention that, for the sequence $\left\{\left(c_{L_{n}}, u_{L_{n}}\right)\right\}_{n \in \mathbb{N}}$ which we consider in Theorem 2.3, we have $\underline{c}=2 \sqrt{\left.\alpha_{1}<\mu\right\rangle_{A}}$ (see Step 1) and $\bar{c}=\sup _{n \in \mathbb{N}} c_{L_{n}}$.

Since $\varphi_{L}(-\infty, x)=0$ and $\varphi_{L}(+\infty, x)=p(x)$ in $C^{2}(\mathbb{R})$ (for each $L>0$ ), it follows then that $\nabla_{s, x} \varphi_{L}(-\infty, x)=0, \partial_{x} \varphi_{L}(+\infty, x)=p_{L}^{\prime}(x)$, and $\partial_{s} \varphi_{L}(+\infty, x)=0$ uniformly in $x \in \mathbb{R}$. Integrating (3.19) by parts over $\mathbb{R} \times[-k L, k L]$ (where $L>0$ and $k \in \mathbb{N}$ ) and using the $L$-periodicity of $\varphi_{L}$ with respect to $x$, we then get

$$
\iint_{\mathbb{R} \times(-k L, k L)} f\left(\frac{x}{L}, \varphi_{L}(s, x)\right) d s d x=c_{L} \int_{-k L}^{k L} p_{L}(x) d x-\int_{-k L}^{k L} a_{L} p_{L}^{\prime}(x) d x,
$$

or equivalently

$$
\iint_{\mathbb{R} \times(-k L, k L)} f\left(\frac{x}{L}, u_{L}(t, x)\right) d t d x=\int_{-k L}^{k L} p_{L}(x) d x-\frac{1}{c_{L}} \int_{-k L}^{k L} a_{L} p_{L}^{\prime}(x) d x .
$$

As in the proof of Lemma 2.1, we take any compact interval $\mathcal{K} \subset \mathbb{R}$ and we define $k_{L} \in \mathbb{N}$ as in (3.3). We apply (3.20) for $k=k_{L}$. Having $0<u_{L}(t, x)<p_{L} \leq M$ for all $(t, x) \in \mathbb{R} \times \mathbb{R}$ and owing to (1.3), (1.6), and (3.4), one then gets

$$
0<\iint_{\mathbb{R} \times \mathcal{K}} f\left(\frac{x}{L}, u_{L}(t, x)\right) d t d x \leq C_{2}(\mathcal{K})
$$


for all $0<L<1$, where

$$
C_{2}(\mathcal{K}):=M(|\mathcal{K}|+2)+\frac{\alpha_{2}}{\underline{c}} \sqrt{C(\mathcal{K})} \sqrt{|\mathcal{K}|+2}
$$

is a constant independent of $L$.

Multiplying (3.19) by $\varphi_{L}$ and integrating by parts over $\mathbb{R} \times(-k L, k L)$, we obtain

$$
\begin{aligned}
\frac{c_{L}}{2} \int_{-k L}^{k L} p_{L}^{2}(x) d x=- & \iint_{\mathbb{R} \times(-k L, k L)}\left[a_{L}\left(\frac{\partial \varphi_{L}}{\partial x}\right)^{2}+a_{L}\left(\frac{\partial \varphi_{L}}{\partial s}\right)^{2}+2 a_{L} \frac{\partial \varphi_{L}}{\partial x} \frac{\partial \varphi_{L}}{\partial s}\right] d s d x \\
& +\int_{-k L}^{k L} a_{L} p_{L}^{\prime} p_{L} d x+\iint_{\mathbb{R} \times(-k L, k L)} f\left(\frac{x}{L}, \varphi_{L}(s, x)\right) \varphi_{L} \\
= & -\iint_{\mathbb{R} \times(-k L, k L)} a_{L}\left(\frac{\partial u_{L}}{\partial x}\right)^{2} d t d x+\int_{-k L}^{k L} a_{L} p_{L}^{\prime} p_{L} d x \\
& +\iint_{\mathbb{R} \times(-k L, k L)} f\left(\frac{x}{L}, u_{L}(t, x)\right) u_{L} d t d x .
\end{aligned}
$$

Notice that the last integral in (3.22) converges because of (3.21) and $0 \leq$ $f\left(x / L, u_{L}\right) u_{L} \leq M f\left(x / L, u_{L}\right)$ in $\mathbb{R} \times \mathbb{R}$. Moreover,

$$
\forall L>0,\left|\int_{-k L}^{k L} a_{L} p_{L}^{\prime} p_{L} d x\right| \leq \alpha_{2} M(2 k L)^{1 / 2}\left(\int_{-k L}^{k L}{p_{L}^{\prime}}^{2}\right)^{1 / 2}
$$

Consequently,

$$
\iint_{\mathbb{R} \times \mathcal{K}}\left(\frac{\partial u_{L}}{\partial x}\right)^{2} d t d x \leq C_{3}(\mathcal{K})
$$

where $C_{3}(\mathcal{K}):=\frac{1}{\alpha_{1}}\left[M C_{2}(\mathcal{K})+\alpha_{2} M(2+|\mathcal{K}|)^{1 / 2} \sqrt{C(\mathcal{K})}\right]$ is independent of $L$.

Now, we multiply (3.19) by $\partial_{s} \varphi_{L}$ and we integrate by parts over $\mathbb{R} \times(-k L, k L)$. We notice that, from the $L$-periodicity with respect to $x$ of the function $\varphi_{L}$ and its derivatives together with the limits of $\partial_{s} \varphi_{L}$ and $\partial_{x} \varphi_{L}$ as $s \rightarrow \pm \infty$, we have

$$
\iint_{\mathbb{R} \times(-k L, k L)} \partial_{x}\left(a_{L} \partial_{x} \varphi_{L}\right) \partial_{s} \varphi_{L}=-\frac{1}{2} \iint_{\mathbb{R} \times(-k L, k L)} \partial_{s}\left(a_{L}\left(\partial_{x} \varphi_{L}\right)^{2}\right)=-\frac{1}{2} \int_{-k L}^{k L} a_{L} p_{L}^{\prime^{2}} d x,
$$

while

$$
\iint_{\mathbb{R} \times(-k L, k L)} \partial_{s} \varphi_{L} \partial_{x}\left(a_{L} \partial_{s} \varphi_{L}\right)+\partial_{s} \varphi_{L} \partial_{s}\left(a_{L} \partial_{x} \varphi_{L}\right)=0
$$

Thus,

$$
c_{L} \iint_{\mathbb{R} \times(-k L, k L)}\left(\frac{\partial \varphi_{L}}{\partial s}\right)^{2}=-\frac{1}{2} \int_{-k L}^{k L} a_{L} p_{L}^{2} d x+\int_{-k L}^{k L} F\left(\frac{x}{L}, p_{L}(x)\right) d x,
$$


where $F(y, s)=\int_{0}^{s} f(y, \tau) d \tau$. Hence,

$$
\iint_{\mathbb{R} \times(-k L, k L)}\left(\frac{\partial u_{L}}{\partial t}\right)^{2} d t d x \leq c_{L} \int_{-k L}^{k L} F\left(\frac{x}{L}, p_{L}(x)\right) d x \leq \bar{c} \int_{-k L}^{k L} F\left(\frac{x}{L}, p_{L}(x)\right) d x .
$$

Consequently, for all $0<L<1$,

$$
\iint_{\mathbb{R} \times \mathcal{K}}\left(\frac{\partial u_{L}}{\partial t}\right)^{2} d t d x \leq \iint_{\mathbb{R} \times\left(-k_{L} L, k_{L} L\right)}\left(\frac{\partial u_{L}}{\partial t}\right)^{2} d t d x \leq C_{4}(\mathcal{K}),
$$

where $C_{4}(\mathcal{K}):=\bar{c}(2+|\mathcal{K}|) \max _{(x, s) \in \mathbb{R} \times[0, M]} F(x, s)$ is a positive constant which is independent of $L$ and depending only on the compact $\mathcal{K}$.

Denote

$$
v_{L}(t, x)=a_{L}(x) \frac{\partial u_{L}}{\partial x}(t, x) \text { and } w_{L}(t, x)=\frac{\partial u_{L}}{\partial t}(t, x) \text { in } \mathbb{R} \times \mathbb{R} .
$$

As already underlined, it follows from [5] ( and [1] in the case $p_{L} \equiv 1$ ) that $w_{L}=\frac{\partial u_{L}}{\partial t}>0$ in $\mathbb{R} \times \mathbb{R}$ for each $L>0$. We shall now establish some estimates (independent of $L$ ) for the functions $v_{L}$ and $w_{L}$, in order to pass to the limit as $L \rightarrow 0^{+}$.

We first recall that for each $L>0, \varphi_{L} \rightarrow p_{L}(x)$ in $C^{2}(\mathbb{R})$ as $s \rightarrow+\infty$ and $\varphi_{L} \rightarrow 0$ in $C^{2}(\mathbb{R})$ as $s \rightarrow-\infty$ (this was proved by construction of pulsating traveling fronts in [5] for example). Now, using the relation between $u_{L}$ and $\varphi_{L}$, one concludes that for each $L>0, u_{L}(-\infty, x)=0$ and $u_{L}(+\infty, x)=p_{L}(x)$ locally in $x$, and $w_{L}( \pm \infty, x)=0$ locally in $x$. On the other hand, (3.25) yields that for each compact $\mathcal{K}$ and for each $L$, $\left\|w_{L}\right\|_{L^{2}(\mathbb{R} \times \mathcal{K})} \leq \sqrt{C(\mathcal{K})}$. Now, we differentiate (3.19) with respect to $t$ (actually, from the regularity of $f$, the function $w_{L}$ is of class $C^{2}$ with respect to $x$ ). There holds

$$
\frac{\partial w_{L}}{\partial t}=\frac{\partial}{\partial x}\left(a_{L}(x) \frac{\partial w_{L}}{\partial x}\right)+f_{u}^{\prime}\left(\frac{x}{L}, u_{L}\right) w_{L} \text { in } \mathbb{R} \times \mathbb{R} .
$$

Multiply the above equation by $w_{L}$ and integrate by parts over $\mathbb{R} \times(-k L, k L)$. From (1.3), (3.24), and the fact that $0<u_{L} \leq M$, it follows that

$$
\iint_{\mathbb{R} \times(-k L, k L)}\left(\frac{\partial w_{L}}{\partial x}\right)^{2} d t d x \leq \frac{2 k L \eta c_{L}}{\alpha_{1}},
$$

where $\eta$ is the positive constant defined by

$$
\eta=\max _{(x, u) \in \mathbb{R} \times[0, M]}\left|f_{u}^{\prime}(x, u)\right| \max _{x \in \mathbb{R}}|F(x, M)| \geq \frac{1}{2 k L} \iint_{\mathbb{R} \times(-k L, k L)} f_{u}^{\prime}\left(\frac{x}{L}, u_{L}\right) w_{L}^{2} d t d x>0 .
$$

Then, for each compact $\mathcal{K} \subset \mathbb{R}$, there exists a constant $C^{\prime}(\mathcal{K})>0$ depending only on $\mathcal{K}$ such that

$$
\forall 0<L<1, \iint_{\mathbb{R} \times \mathcal{K}}\left(\frac{\partial w_{L}}{\partial x}\right)^{2} d t d x \leq C^{\prime}(\mathcal{K}) .
$$

We pass now to the family $\left\{v_{L}\right\}_{L}$. Actually, $v_{L}=a_{L} \frac{\partial u_{L}}{\partial x}$ and $0<\alpha_{1} \leq a_{L} \leq \alpha_{2}$ for each $L>0$. Thus, (3.23) yields that for each compact interval $\mathcal{K}$ of $\mathbb{R}$ and for each $0<L<1$, $\left\|v_{L}\right\|_{L^{2}(\mathbb{R} \times \mathcal{K})} \leq \alpha_{2} \sqrt{C_{3}(\mathcal{K})}$. Furthermore, (1.11) implies that

$$
\forall L>0, \frac{\partial v_{L}}{\partial x}=\frac{\partial u_{L}}{\partial t}-f\left(\frac{x}{L}, u_{L}\right) \text { in } \mathbb{R} \times \mathbb{R},
$$


while $0 \leq f\left(x / L, u_{L}(t, x)\right) \leq \kappa$ in $\mathbb{R} \times \mathbb{R}$ where $\kappa=\max _{\mathbb{R} \times[0, M]} f(x, u)>0$ is independent of $L$. Together with (3.25), one concludes that any family $\left\{\partial v_{L} / \partial x\right\}_{0<L<1}$ is bounded in $L_{l o c}^{2}(\mathbb{R} \times \mathbb{R})$ by a constant independent of $L$. On the other hand,

$$
\forall L>0, \frac{\partial v_{L}}{\partial t}=a_{L} \frac{\partial^{2} u_{L}}{\partial t \partial x}=a_{L} \frac{\partial w_{L}}{\partial x} \text { in } \mathbb{R} \times \mathbb{R} .
$$

Owing to (1.3) and (3.26), any family $\left\{\frac{\partial v_{L}}{\partial t}\right\}_{0<L<1}$ is bounded in $L_{l o c}^{2}(\mathbb{R} \times \mathbb{R})$.

Step 4: Passage to the limit as $n \rightarrow+\infty\left(L \rightarrow 0^{+}\right)$. In this step, we consider the sequence $\left\{L_{n}\right\}_{n \in \mathbb{N}}$ of Theorem 2.3 which is in $(0,1)$ and which tends to 0 as $n \rightarrow+\infty$. As a consequence of the previous step, $\left\{v_{L_{n}}\right\}_{n \in \mathbb{N}}$ is bounded in $H_{l o c}^{1}(\mathbb{R} \times \mathbb{R})$. The estimates (3.23) and (3.25) imply that the sequence $\left\{u_{L_{n}}\right\}_{n \in \mathbb{N}}$ is bounded in $H_{\text {loc }}^{1}(\mathbb{R} \times \mathbb{R})$. Thus, there exist $u_{0}$ and $v_{0}$ in $H_{\text {loc }}^{1}(\mathbb{R} \times \mathbb{R})$ such that, up to extraction of a subsequence, $u_{L_{n}} \rightarrow u_{0}, v_{L_{n}} \rightarrow v_{0}$ strongly in $L_{l o c}^{2}(\mathbb{R} \times \mathbb{R})$ and almost everywhere in $\mathbb{R} \times \mathbb{R}$,

$$
\left(\frac{\partial u_{L_{n}}}{\partial t}, \frac{\partial u_{L_{n}}}{\partial x}\right) \rightarrow\left(\frac{\partial u_{0}}{\partial t}, \frac{\partial u_{0}}{\partial x}\right) \text { weakly in } L_{l o c}^{2}(\mathbb{R} \times \mathbb{R}),
$$

and

$$
\left(\frac{\partial v_{L_{n}}}{\partial t}, \frac{\partial v_{L_{n}}}{\partial x}\right) \rightarrow\left(\frac{\partial v_{0}}{\partial t}, \frac{\partial v_{0}}{\partial x}\right) \text { weakly in } L_{l o c}^{2}(\mathbb{R} \times \mathbb{R})
$$

as $n \rightarrow+\infty$. However, $\left.a_{L_{n}}^{-1} \rightarrow<a^{-1}\right\rangle_{A}=\langle a\rangle_{H}^{-1}$ in $L^{\infty}(\mathbb{R})$ weak-* as $n \rightarrow+\infty$. Thus,

$$
\frac{\partial u_{L_{n}}}{\partial x}=\frac{v_{L_{n}}}{a_{L_{n}}} \rightarrow \frac{v_{0}}{<a>_{H}} \text { weakly in } L_{l o c}^{2}(\mathbb{R} \times \mathbb{R}) \text { as } n \rightarrow+\infty .
$$

By uniqueness of the limit, one gets $v_{0}=<a>_{H} \frac{\partial u_{0}}{\partial x}$. On the other hand, $f\left(\frac{x}{L_{n}}, u_{L_{n}}\right) \rightarrow$ $g\left(u_{0}\right)$ in $L^{\infty}(\mathbb{R} \times \mathbb{R})$ weak-* as $n \rightarrow+\infty$. Passing to the limit as $n \rightarrow+\infty$ in the first equation of (1.11) with $L=L_{n}$ implies that $u_{0}$ is a weak solution of the equation

$$
\frac{\partial u_{0}}{\partial t}=\frac{\partial v_{0}}{\partial x}+g\left(u_{0}\right)=<a>_{H} \frac{\partial^{2} u_{0}}{\partial x^{2}}+g\left(u_{0}\right) \text { in } \mathcal{D}^{\prime}(\mathbb{R} \times \mathbb{R}) .
$$

From parabolic regularity, the function $u_{0}$ is then a classical solution of the homogeneous equation

$$
\frac{\partial u_{0}}{\partial t}=<a>_{H} \frac{\partial^{2} u_{0}}{\partial x^{2}}+g\left(u_{0}\right) \text { in } \mathbb{R} \times \mathbb{R}
$$

such that $0 \leq u_{0} \leq p_{0}$ and $\frac{\partial u_{0}}{\partial t} \geq 0$ in $\mathbb{R} \times \mathbb{R}$. Lastly, it follows directly from the normalization (3.17) that

$$
\iint_{(0,1)^{2}} u_{0}(t, x) d t d x=\frac{p_{0}}{2}
$$

On the other hand, it follows from the second equation of (1.11) that

$$
\forall \gamma \in \mathbb{R}, \quad u_{0}\left(t+\frac{\gamma}{c}, x\right)=u_{0}(t, x+\gamma) \text { in } \mathbb{R} \times \mathbb{R},
$$


where $c=\lim _{n \rightarrow+\infty} c_{L_{n}}>0$. In other words, $u_{0}(t, x)=U_{0}(x+c t)$, where $U_{0}$ is a classical solution of the equation

$$
c U_{0}^{\prime}=<a>_{H} U_{0}^{\prime \prime}+<\mu>_{A} g\left(U_{0}\right) \text { in } \mathbb{R},
$$

that satisfies $U_{0}^{\prime} \geq 0$ in $\mathbb{R}, 0 \leq U_{0}(s) \leq p_{0}$ for all $s \in \mathbb{R}$.

Standard elliptic estimates on (3.28) imply that $U_{0}$ converges as $s \rightarrow \pm \infty$ in $C_{l o c}^{2}(\mathbb{R})$ to two constants $U_{0}^{ \pm} \in\left[0, p_{0}\right]$ such that $g\left(U_{0}^{ \pm}\right)=0$.

Thanks to the normalization $(3.27), u_{0}(t, x)=U_{0}(x+c t)$ satisfies the normalization

$$
\int_{0}^{1}\left(\int_{c s}^{c s+1} U_{0}(\tau) d \tau\right) d s=\frac{p_{0}}{2}
$$

The monotonicity of $U_{0}$ and the nature of the function $g$ imply that $U_{0}^{-}=0$ and $U_{0}^{+}=p_{0}$. Together with the normalization (3.29), one concludes that $U_{0}$ is the unique traveling front for the homogenized Equation (3.28) with a speed $c$ and limiting conditions 0 and $p_{0}$ at infinity. We mention that, since the minimal speed for the problem (3.28) is equal to $2 \sqrt{\langle a\rangle_{H} g^{\prime}(0)}=2 \sqrt{\langle a\rangle_{H}\langle\mu\rangle_{A}}$, one can review that $c \geq 2 \sqrt{\left.\langle a\rangle_{H}<\mu\right\rangle_{A}}$, which was proved by other tools in [10]. Eventually, the proof of Theorem 2.3 is complete.

Acknowledgments. The author would like to thank the Referees for their valuable suggestions and comments which helped in improving the presentation of Theorem 2.3.

\section{REFERENCES}

[1] H. Berestycki and F. Hamel, Front propagation in periodic excitable media, Commun. Pure Appl. Math., 55, 949-1032, 2002.

[2] H. Berestycki, F. Hamel, and N. Nadirashvili, The principal eigenvalue of elliptic operators with large drift and applications to nonlinear propagation phenomena, Commun. Math. Phys., 253, 451-480, 2005.

[3] H. Berestycki, F. Hamel, and N. Nadirashvili, The speed of propagation for KPP type problems. I-Periodic framework, J. Eur. Math. Soc., 7, 173-213, 2005.

[4] H. Berestycki, F. Hamel, and L. Roques, Analysis of the periodically fragmented environment model: I-Species persistence, J. Math. Biol., 5, 75-113, 2005.

[5] H. Berestycki, F. Hamel, and L. Roques, Analysis of the periodically fragmented environment model: II-Biological invasions and pulsating traveling fronts, J. Math. Pures Appl., 84, 11011146, 2005.

[6] L.A. Caffarelli, K.A. Lee, and A. Mellet, Singular limit and homogenization for flame propagation in periodic excitable media, Arch. Ration. Mech. Anal., 172, 153-190, 2004.

[7] L.A. Caffarelli, K.A. Lee, and A. Mellet, Homogenization and flame propagation in periodic excitable media: The asymptotic speed of propagation, Commun. Pure Appl. Math., 59, 501525, 2006.

[8] M. El Smaily, Pulsating traveling fronts: Asymptotics and homogenization regimes, European J. Appl. Math., 19, 393-434, 2008.

[9] M. El Smaily, Min-Max formulae for the speeds of pulsating traveling fronts in periodic excitable media, Annali di Matematica Pura ed Applicata, 189(1), 47-66, 2010.

[10] M. El Smaily, F. Hamel, and L. Roques, Homogenization and influence of fragmentation in a biological invasion model, Discrete Contin. Dyn. Syst. Series A, in the special issue in honour of Professor Masayasu Mimura, 25(1), 321-342, 2009.

[11] M. El Smaily and S. Kirsch, The speed of propagation for KPP reaction-diffusion equations within large drift, Adv. Diff. Equ., 16(3-4), 361-400, 2011.

[12] R.A. Fisher, The wave of advance of advantageous genes, Ann. Eugenics, 7, 355-369, 1937.

[13] F. Hamel and L. Roques, Mathematical analysis of the optimal habitat configurations for species persistence, Math. Biosciences, 210, 34-59, 2007. 
[14] F. Hamel and L. Roques, Uniqueness and stability properties of monostable pulsating fronts, J. European Math. Soc., 13, 345-390, 2011.

[15] S. Heinze, Large convection limits for KPP fronts, Max-Planck Institut für Mathimatik in den Naturwissenschaften, Leipzig, preprint 21/(2005).

[16] N. Kinezaki, K. Kawasaki, F. Takasu, and N. Shigesada, Modeling biological invasion into periodically fragmented environments, Theor. Popul. Biol., 64, 291-302, 2003.

[17] A.N. Kolmogorov, I.G. Petrovsky, and N.S. Piskunov, Étude de l'équation de la diffusion avec croissance de la quantité de matière et son application à un problème biologique, Bull. Univ. État Moscou (Bjul. Moskowskogo Gos. Univ.), Sér. Inter., A1, 1-26, 1937.

[18] X. Liang, X. Lin, and H. Matano, Maximizing the spreading speed of KPP fronts in twodimensional stratified media, preprint arXiv:1004.0572.

[19] X. Liang, X. Lin, and H. Matano, A variational problem associated with the minimal speed of traveling waves for spatially periodic reaction-diffusion equations, Trans. Amer. Math. Soc., 362, 5605-5633, 2010.

[20] G. Nadin, Traveling fronts in space-time periodic media, J. Math. Pures Appl. 92, 232-262, 2009.

[21] J. Nolen, M. Rudd, and J. Xin, Existence of KPP fronts in spatially-temporally periodic advection and variational principle for propagation speeds, Dyn. Part. Diff. Eqs., 2, 1-24, 2005.

[22] J. Nolen, and J. Xin, Existence of KPP type fronts in space-time periodic shear flows and a study of minimal speeds based on variational principle, Disc. Cont. Dyn. Syst., 13, 1217-1234, 2005.

[23] L. Ryzhik and A. Zlatoš, KPP pulsating front speed-up by flows, Commun. Math. Sci., 5, 575$593,2007$.

[24] N. Shigesada, K. Kawasaki, and E. Teramoto, Traveling periodic waves in heterogeneous environments, Theor. Popul. Biol., 30, 143-160, 1986.

[25] N. Shigesada, K. Kawasaki, and E. Teramoto, The speeds of traveling frontal waves in heterogeneous environments, in Mathematical Topics in Population Biology, Morphogenesis and Neurosciences, Teramoto E., Yamaguti M. (eds), Lecture Notes in Biomathematics, Springer, $71,87-97,1987$.

[26] H.F. Weinberger, On spreading speeds and traveling waves for growth and migration in periodic habitat, J. Math. Biol., 45, 511-548, 2002.

[27] X. Xin, Existence and uniqueness of traveling waves in a reaction-diffusion equation with combustion nonlinearity, Indiana Univ. Math. J., 40, 985-1008, 1991.

[28] X. Xin, Existence and stability of traveling waves in periodic media governed by a bistable nonlinearity, J. Dyn. Diff. Eqs., 3, 541-573, 1991.

[29] X. Xin, Existence of planar flame fronts in convective-diffusive periodic media, Arch. Ration. Mech. Anal., 121, 205-233, 1992.

[30] J.X. Xin, Analysis and modeling of front propagation in heterogeneous media, SIAM Review, 42, 161-230, 2000.

[31] A. Zlatoš, Sharp asymptotics for KPP pulsating front speed-up and diffusion enhancement by flows, Arch. Ration. Mech. Anal., 195, 441-453, 2010. 